\title{
RESEARCH
}

Open Access

\section{NT-4 attenuates neuroinflammation via TrkB/PI3K/FoxO1 pathway after germinal matrix hemorrhage in neonatal rats}

\author{
Tianyi Wang ${ }^{1,2+}$, Junyi Zhang ${ }^{1,2+} \mathbb{D}$, Peng Li ${ }^{1}$, Yan Ding ${ }^{1}$, Jiping Tang ${ }^{1}$, Gang Chen ${ }^{2^{*}}$ and John H. Zhang ${ }^{*^{*}}$
}

\begin{abstract}
Background: Neuroinflammation plays an important role in pathogenesis of germinal matrix hemorrhage (GMH). Neurotrophin-4 (NT-4) is a member of the neurotrophin family and interacts with the tropomyosin receptor kinase B (TrkB). NT-4 has been shown to confer neuroprotective effects following cerebral ischemia. We aimed to investigate the neuroprotective function of NT-4-TrkB signaling, as well as its downstream signaling cascade phosphatidylinositol-3-kinases (PI3K)/protein kinase B (Akt)/forkhead box protein O1 (FoxO1), following GMH in neonatal rats.
\end{abstract}

Methods: GMH was induced by intraparenchymal injection of bacterial collagenase $(0.3 \mathrm{U})$ in P7 rat pups. A total of 163 pups were used in this study. Recombinant human NT-4 was administered intranasally at $1 \mathrm{~h}$ after the collagenase injection. The selective TrkB antagonist ANA-12, selective PI3K inhibitor LY294002, and FoxO1 activating CRISPR were administered intracerebroventricularly at $24 \mathrm{~h}$ prior to NT-4 treatment to investigate the underlying mechanism. Short-term and long-term neurobehavioral assessments, immunofluorescence staining, Nissl's staining, and Western blot were performed.

Results: Expression of phosphorylated TrkB increased after $\mathrm{GMH}$, reaching the peak level at day 3 after hemorrhage. TrkB receptors were observed on neurons, microglia, and astrocytes. The administration of rh-NT-4 induced phosphorylation of TrkB, expression of PI3K, and phosphorylation of Akt. Meanwhile, it decreased FoxO1 and IL-6 levels. Selective inhibition of TrkB/PI3K/Akt signaling in microglia increased the expression levels of FoxO1 and proinflammatory cytokines. FoxO1 activating CRISPR increased the expression of IL-6, suggesting that FoxO1 might be a potential inducer of pro-inflammatory factors. These results suggested that PI3K/Akt/FoxO1 signaling may be the downstream pathway of activation of TrkB. The rat pups treated with rh-NT-4 performed better than vehicle-treated animals in both short-term and long-term behavioral tests.

(Continued on next page)

\footnotetext{
*Correspondence: nju_neurosurgery@163.com; johnzhang3910@yahoo.com

†Tianyi Wang and Junyi Zhang contributed equally to this work.

${ }^{2}$ Department of Neurosurgery, The First Affiliated Hospital of Soochow

University, Suzhou 215000, China

'Department of Physiology and Pharmacology, Loma Linda University,

School of Medicine, 11041 Campus Street, Loma Linda, CA 92350, USA
}

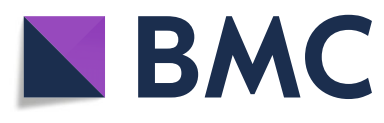

(c) The Author(s). 2020 Open Access This article is licensed under a Creative Commons Attribution 4.0 International License, which permits use, sharing, adaptation, distribution and reproduction in any medium or format, as long as you give appropriate credit to the original author(s) and the source, provide a link to the Creative Commons licence, and indicate if changes were made. The images or other third party material in this article are included in the article's Creative Commons licence, unless indicated otherwise in a credit line to the material. If material is not included in the article's Creative Commons licence and your intended use is not permitted by statutory regulation or exceeds the permitted use, you will need to obtain permission directly from the copyright holder. To view a copy of this licence, visit http://creativecommons.org/licenses/by/4.0/ The Creative Commons Public Domain Dedication waiver (http://creativecommons.org/publicdomain/zero/1.0/) applies to the data made available in this article, unless otherwise stated in a credit line to the data. 


\begin{abstract}
(Continued from previous page)
Conclusion: These data showed that rh-NT-4 reduced the expression levels of pro-inflammatory cytokines, improved neurological function, attenuated neuroinflammation, and thereby mitigated post-hemorrhagic hydrocephalus after GMH by TrkB/PI3K/Akt/FoxO1 pathway. These results indicated that rh-NT-4 could be a promising therapeutic strategy to ameliorate neuroinflammation and hydrocephalus after $\mathrm{GMH}$ or other similar brain injuries.
\end{abstract}

Keywords: Germinal matrix hemorrhage, Neurotrophin-4, TrkB, Neuroinflammation, hydrocephalus

\section{Background}

Germinal matrix hemorrhage (GMH) is the most common and devastating neurological injury of premature infants. It has been reported that GMH affects 7-30\% of premature neonates. Depending on the grade of the hemorrhage, $25-80 \%$ of them will develop hydrocephalus $[5,6]$. After the hemorrhage occurs, the inflammatory cascade will be activated to amplify the damage of brain tissues and it may lead to developmental delays, mental retardation, cerebral palsy, and hydrocephalus [19]. According to previous studies, suppressing proinflammatory factors could be beneficial in attenuating the GMH-induced brain injury [40]. Microglia, accounting for $10-15 \%$ of cells in the brain, function as the first and primary form of active immune cells in the central nervous system (CNS) [4]. Traditionally, microglia play a critical role in the immune response after GMH and are considered injurious in the post-hemorrhagic brain due to the production of inflammatory cytokines [37, 40]. Therefore, suppression of microglia activation could have a beneficial effect after GMH. TrkB is the highaffinity receptor for several neurotrophins, such as BDNF, NT-4, and NT-3[3, 15, 21]. Its expression level is relatively high in the brain tissues. Previous studies showed that the activation of $\operatorname{TrkB}$ could attenuate neurological deficits after hemorrhagic stroke [27, 36]. Neurotrophin-4(NT-4) is a neurotrophic factor that signals mainly through the tyrosine receptor kinase $B$ (TrkB) [34]. It has been reported that the increase of NT-4 reduced the infarct area after ischemic stroke [10, $13,14]$. However, the effect of NT-4 in GMH is still unclear. In this study, we are going to explore the antiinflammatory effect of NT-4/TrkB/PI3K/FoxO1 signaling pathway after GMH in neonatal rats [12, 29, 32].

\section{Materials and methods}

\section{Animals}

All experimental procedures were approved by the Institutional Animal Care and Use Committee at Loma Linda University. All studies were conducted in accordance with the United States Public Health Service's Policy on Humane Care and Use of Laboratory Animals and reported according to the ARRIVE guidelines. One hundred and sixty-three P7 Sprague-Dawley neonatal pups (weight $=12-14 \mathrm{~g}$, Harlan, Livermore, CA) were randomly divided into sham and GMH groups (Supplementary Fig. 1). All pups were kept in rooms with controlled temperature and 12-h light/dark cycles, and given ad libitum access to food and water.

\section{Experimental design \\ Experiment 1}

To determine the time course of endogenous NT-4, TrkB and phosphorylated TrkB expression profile after $\mathrm{GMH}$, thirty-six P7 rat pups were randomly divided into six groups: naive $(n=6), 12 \mathrm{~h}$ after GMH $(n=6), 1$ day after GMH $(n=6), 3$ days after GMH $(n=6), 5$ days after GMH $(n=6), 7$ days after GMH $(n=6)$. Western blot analysis was performed to detect their expression in whole brain tissues of each group.

\section{Experiment 2}

The cellular localization of TrkB was evaluated by immunofluorescence staining to co-localize TrkB with ionized calcium-binding adaptor molecule 1 (Iba-1), neuronal-specific nuclear protein (NeuN), and glia fibrillary acidic protein (GFAP) respectively at 3 days after GMH $(n=6)$. Anti-human NT-4 antibody was used in this part. The concentration of exogenous NT-4 was analyzed by Western blot. A total of eighteen P7 rat pups were randomly divided into 3 groups (sham, GMH + vehicle, GMH + rh-NT-4). The whole brains were collected for Western blot at $6 \mathrm{~h}$ after GMH.

\section{Experiment 3}

The outcomes of rh-NT-4 treatment were assessed during the first 3 days and during day 21 and day 28 after $\mathrm{GMH}$. The pups were randomly divided into 5 groups: sham, GMH + vehicle (5\% DMSO), GMH + rh-NT-4 $(0.03 \mathrm{mg} / \mathrm{kg}), \mathrm{GMH}+\mathrm{rh}-\mathrm{NT}-4(0.1 \mathrm{mg} / \mathrm{kg})$, and GMH + rh-NT-4 $(0.3 \mathrm{mg} / \mathrm{kg})$. Rh-NT-4 was dissolved in $5 \%$ DMSO and administered intranasally at a total volume of $2 \mu \mathrm{l}$ at $1 \mathrm{~h}, 25 \mathrm{~h}$, and $49 \mathrm{~h}$ post GMH. Short-term behavioral tests (negative geotaxis and body righting reflex) were performed to determine the optimal dosage of rhNT-4 for long-term outcome studies. The sample size was then increased to 12 for each group (sham, GMH + vehicle, GMH + rh-NT-4) for long-term behavioral tests 
(rotarod test, foot fault test, and Morris water maze). The whole brains were collected for Nissl staining at 28 days after GMH.

\section{Experiment 4}

To explore the underlying mechanisms of the rh-NT-4mediated anti-inflammatory effects after GMH (Supplementary Fig. 2), the selective TrkB antagonist ANA-12 was administered intraperitoneally at $1 \mathrm{~h}$ before GMH induction. The specific PI3K inhibitor LY-294002 was administered intracerebroventricularly at $1 \mathrm{~h}$ before GMH induction. The FoxO1 activating CRISPR was administered intracerebroventricularly at $24 \mathrm{~h}$ before $\mathrm{GMH}$ induction, and followed with rh-NT-4 $(0.1 \mathrm{mg} / \mathrm{kg})$ treatment after GMH. Rat pups were divided into nine groups: sham $(n=6), \mathrm{GMH}+$ vehicle $(n=6), \mathrm{GMH}+$ rh-NT-4 $(n=6), \mathrm{GMH}+$ rh-NT-4 + ANA-12 $(n=6)$, GMH + rh-NT- $4+$ ANA-12 control $(n=6)$, GMH + rhNT-4 + LY294002 $(n=6)$, GMH + rh-NT-4 + LY294002 control $(n=6)$, GMH + rh-NT-4 + FoxO1 CRISPR $(n=6), \mathrm{GMH}+$ rh-NT-4 + CRISPR control $(n$ $=6)$. Whole-brain tissues were collected on the third day after GMH.

\section{GMH model}

The general procedure for inducing GMH in unsexed P7 rats using collagenase infusion was performed as previously described [23]. The rat pups were anesthetized with $3 \%$ isoflurane. The skin was incised on the longitudinal plane to expose the bregma. A burr hole $(1 \mathrm{~mm})$ was drilled on the skull $(1.6 \mathrm{~mm}$ lateral, $1.5 \mathrm{~mm}$ anterior to the bregma), and a 27 -gage needle was inserted (2.7 $\mathrm{mm}$ deep from the dura) for collagenase ( 0.3 unites of clostridial collagenase VII-S, Sigma-Aldrich, MO) infusion $(3 \mu \mathrm{l} / 3 \mathrm{~min})$ using a $10 \mu \mathrm{l}$ Hamilton syringe (Hamilton Co, Reno, NV, USA) guided by a microinfusion pump (Harvard Apparatus, Holliston, MA). After the infusion, the needle was left at the place for $10 \mathrm{~min}$ to prevent "back leakage" and then was withdrawn at rate of $0.5 \mathrm{~mm} / \mathrm{min}$. Once the needle was removed, bone wax was used to seal the burr hole and the incision site was sutured. Animals were allowed to recover on a $37^{\circ} \mathrm{C}$ heated blanket. Animals were put back with their dams after recovering from anesthesia.

\section{Drug administration}

Recombinant human neurotrophin-4 (Genscript) was dissolved in 5\%DMSO. Pups were assigned to receive rh-NT-4 $(0.03 \mathrm{mg} / \mathrm{kg} /$ day, $0.1 \mathrm{mg} / \mathrm{kg} /$ day, or $0.3 \mathrm{mg} / \mathrm{kg} /$ day) or $5 \%$ DMSO intranasally at $1 \mathrm{~h}$ post GMH and then once a day for all studies [11]. The dosage was based on previous studies [2]. ANA-12 (Selleckchem) is a selective TrkB antagonist. It binds directly to TrkB with high affinity and thereby inhibits the downstream signaling of TrkB. ANA-12 $(0.5 \mathrm{mg} / \mathrm{kg})$ was dissolved in $5 \% \mathrm{DMSO}$ at $50 \mathrm{mmol} / \mathrm{L}$ and administered intraperitoneally at $1 \mathrm{~h}$ prior to GMH induction [16]. LY-294002 (Selleckchem) is the first synthetic molecule known to inhibit PI3K in previous studies. LY-294002 was dissolved in $5 \%$ DMSO at $50 \mathrm{mmol} / \mathrm{L}$ and administered intracerebroventricularly at $1 \mathrm{~h}$ prior to $\mathrm{GMH}$ induction [38]. FoxO1 CRISPR (Santa Cruz Biotechnology) was suspended in the plasmid transfection medium and activated with transfection reagent, totaling $2 \mu \mathrm{g}$ per animal. The control scramble CRISPR was prepared following the same steps and a total of $2 \mu \mathrm{g}$ per animal was given intracerebroventricularly [30, 35]. Animals were randomly divided into the following groups: sham group ( $n$ $=6), \mathrm{GMH}+$ vehicle group $(n=6$, i.n.), GMH + rhNT-4 group $(n=6$, i.n.), GMH + rhNT-4 + ANA-12 group ( $n$ $=6$, i.c.v. $), \mathrm{GMH}+\operatorname{rhNT}-4+$ vehicle1 group $(n=6$, i.p.), GMH + rhNT-4 + LY294002 group $(n=6$, i.p.), $\mathrm{GMH}+$ rhNT- $4+$ vehicle 2 group $(n=6$, i.c.v.), GMH + rhNT-4 + FoxO1 CRISPR group ( $n=6$, i.c.v.), GMH + rhNT-4 + control CRISPR group $(n=6$, i.c.v.), (vehicle, $5 \%$ DMSO, intranasal administration; vehicle 1, 5\% DMSO, intraperitoneal injection; vehicle 2, 5\% DMSO, intracerebroventricular injection).

\section{Immunofluorescence staining}

Immunofluorescence staining was performed on fixed frozen brain sections as previously reported $[8,9]$. Ten millimeter-thick slices were permeabilized with $0.3 \%$ Triton X-100 for $20 \mathrm{~min}$ at room temperature, then 5\% normal donkey serum in PBS was used to block the slices for $2 \mathrm{~h}$. After washing with PBS for three times (10 min each), sections were incubated with anti-TrkB (Abcam), anti-ionized calcium binding adapter molecule 1 (Iba- 1, Abcam) at $4{ }^{\circ} \mathrm{C}$ overnight. After that, the slices were incubated for $1 \mathrm{~h}$ with FITC or Texas Redconjugated secondary antibodies at room temperature. Then sections were washed again with PBS for three times $(10 \mathrm{~min}$ each). Slides were covered in mounting media with DAPI. The sections were imaged under a fluorescence microscope (Leica DMi8, Leica Microsystems) equipped with the LASX software.

\section{Nissl staining}

Nissl staining was performed and analyzed as in previous studies [8]. Coronal brain sections (20 mm thick) were respectively dehydrated in $95 \%$ and $70 \%$ FLEX (ThermoFisher) for $1 \mathrm{~min}$, then rinsed in tap water and distilled water for $10 \mathrm{~s}$. Sections were stained with $0.5 \%$ cresyl violet (Sigma-Aldrich) for $1.5 \mathrm{~min}$ and washed in distilled water for $10 \mathrm{~s}$ followed by dehydration with $100 \%$ FLEX and xylene for $1 \mathrm{~min}$ (two times, respectively). The permount was applied before the coverslip was placed. The sections were imaged by a microscope (Olympus- 
BX51). Brain tissue loss and ventricular volume were measured and calculated with ImageJ [5, 22]. Volumes were calculated by the following formula: average (area of coronal section) $\times$ section interval $\times$ number of sections $[17,28]$. Calculations were performed in a blinded fashion.

\section{Western blot}

Brain tissues were collected and stored at $-80{ }^{\circ} \mathrm{C}$ after being perfused with cold PBS (0.1 M, pH 7.4) at $12 \mathrm{~h}, 1$ day, 3 days, 5 days, 7 days for the time course study, and at 3 days for the mechanistic study after GMH induction, respectively. Western blot was performed as previously described [25]. After sample preparation, $50 \mu \mathrm{g}$ protein per sample was loaded onto $10-12 \%$ SDS-PAGE gels, ran for $90 \mathrm{~min}$ at $100 \mathrm{~V}$, and was transferred onto 0.2 $\mathrm{mm}$ or $0.45 \mathrm{~mm}$ nitrocellulose membranes at $100 \mathrm{~V}$ for 120 min (Bio-Rad) [24]. The membranes were blocked for $2 \mathrm{~h}$ in $5 \%$ non-fat milk in Tris-buffered saline with $0.1 \%$ tween 20 , followed by overnight incubation at $4{ }^{\circ} \mathrm{C}$ with the following primary antibodies: anti-human NT-4 (ThermoFisher, USA), anti-NT-4 (Santa Cruz, USA), anti-TrkB (Abcam, USA), anti-pTrkB (ThermoFisher, USA), anti-PI3K(Abcam, USA), anti-Akt (Abcam, USA), anti-pAkt (Abcam, USA), anti-FoxO1(CST, USA), and anti-IL6 (Santa Cruz, USA). The same membranes were probed with actin (Santa Cruz, USA) as internal loading controls. Appropriate secondary antibodies (Santa Cruz, USA) were incubated with membranes for $2 \mathrm{~h}$ at room temperature. Bands were visualized using ECL Plus Chemiluminescence kit (Amersham Biosciences, USA) and quantified through ImageJ 4.0 (Media Cybernetics). And we have the gel scans of all proteins in this study (Supplementary Fig. 3).

\section{Neurobehavioral test}

Neurobehavioral tests were randomly performed by two researchers blinded to the experimental groups designation according to previous studies [23, 40]. Short-term neurobehavioral tests, including righting reflex and negative geotaxis tests, were performed from day 1 to day 3 after GMH. Long-term neurological tests, including foot-fault, rotarod, and water maze, were performed from day 21 to day 28 after GMH.

Righting reflex. Pups were placed in a supine position to record the duration of complete rollover onto four limbs. The maximum duration was $60 \mathrm{~s}$ per trial ( 3 trials/pup/day) and the average values were calculated.

Negative geotaxis. The duration for the pups to rotate to turn $180^{\circ}$ after being placed with the head downward on a $45^{\circ}$ slope was recorded. The maximum duration was $60 \mathrm{~s}$ per trial ( 3 trials/pup/day), and the average values were calculated.
Rotarod test. Pups were placed on a rotarod (Columbus Instruments), and tested at a starting 5 RPM or 10 RPM with acceleration at 2 RPM per $5 \mathrm{~s}$. The latency to fall was recorded, and the maximum recording time was $60 \mathrm{~s}$.

Foot-fault. Foot-fault was recorded as the number of missteps (inaccurately placed a forelimb or hindlimb and fell through one of the openings in the grid) were recorded over $60 \mathrm{~s}$.

Water maze test. Water maze test was a six-day test, including cued tests and hidden tests. The apparatus consisted of a metal pool $(180 \mathrm{~cm}$ in diameter) and a small platform $(11 \mathrm{~cm}$ in diameter) for the pups to climb onto. In the cued test, pups were manually guided to the platform if they could not found the platform, and the location of platform was changed every other trail. In hidden tests, the platform was removed from the pool, and the time spent in probe quadrant was recorded. Data were analyzed by a tracking software (Noldus Ethovision).

\section{Statistical analysis}

Statistical analysis was performed using GraphPad Prism 6 (GraphPad Software). All the data values were presented as mean \pm SD. One-way ANOVA with Dunnet's post hoc test was used for multiple comparisons while a two-tailed Student $t$ test was performed for two-group comparisons. Statistical significance was defined as $p$ value $<0.05$.

\section{Results}

The expression of phosphorylated TrkB increased after GMH

Western blot was performed to test the protein expression of NT-4 and phosphorylated TrkB at $0 \mathrm{~h}, 12 \mathrm{~h}, 1$ day, 3 days, 5 days, and 7 days in the whole brain after GMH. The results showed that the expression of endogenous NT-4 significantly decreased at $12 \mathrm{~h}$ and 1 day, and then increased and maintained at a similar level after 3 days $(p<0.05$, Fig. 1a). The expression of phosphorylated TrkB increased after GMH and peaked at 3 days $(p<0.05$, Fig. $1 b)$.

\section{Intranasal administration of recombinant human NT-4 improved neurological functions during the first 3 days post GMH}

To investigate the effect of recombinant human NT-4 treatment, 3 doses $(0.03,0.1,0.3 \mathrm{mg} / \mathrm{kg})$ of rh-NT-4 were tested with intranasal administrations after GMH. According to the time course study, the expression of endogenous NT-4 decreased at $12 \mathrm{~h}$ and 1 day and then increased at 3 days after GMH. Thus, we used the exogenous rh-NT-4 to supplement the decreased endogenous NT-4 in the first 3 days. Therefore, different doses 

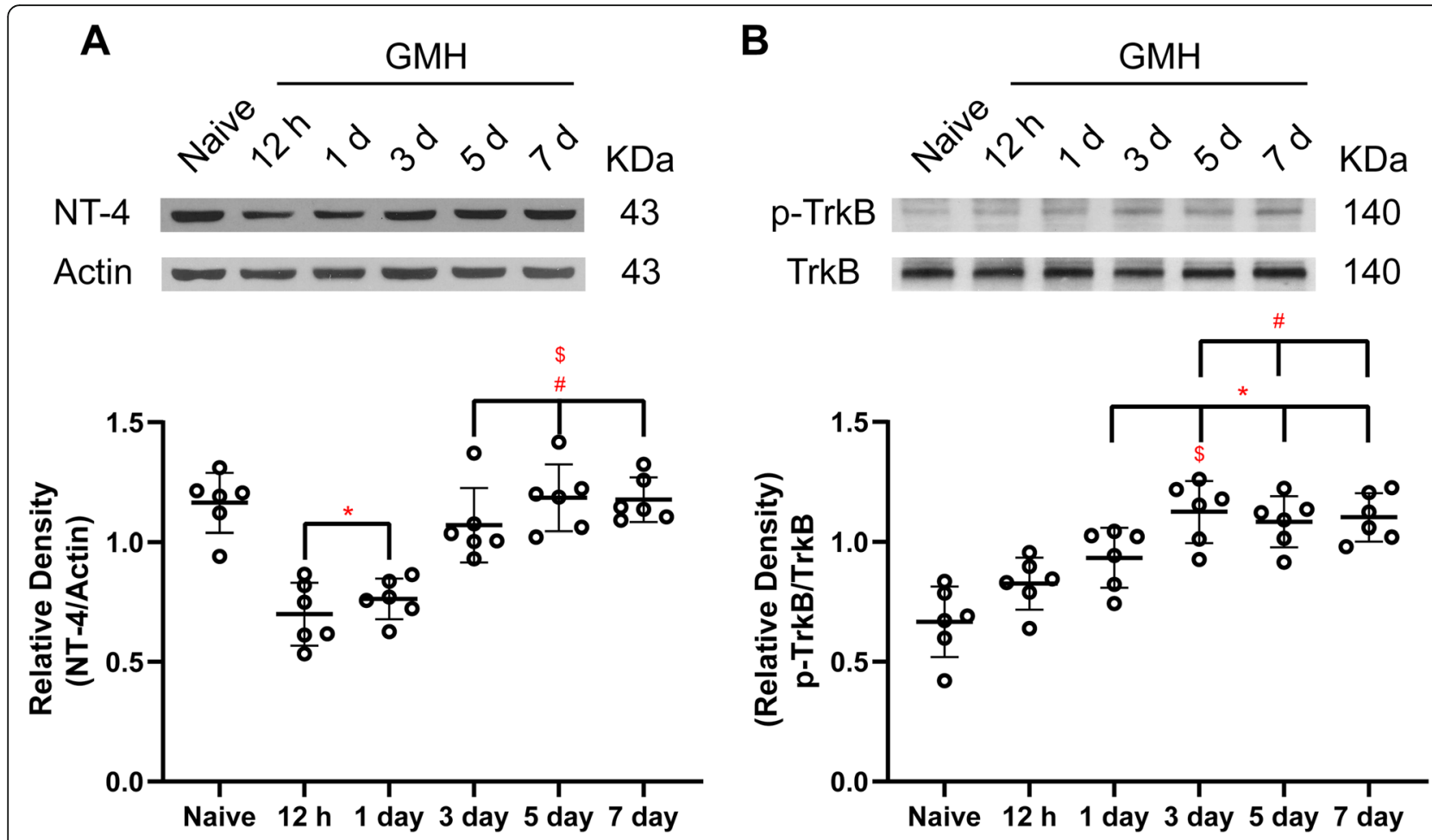

Fig. 1 Expression profile of NT-4 and TrkB after GMH. a Representative Western blot bands of time course and quantitative analyses of NT-4 expression after GMH. b Representative Western blot bands of time course and quantitative analyses of phosphorylated TrkB expression after $\mathrm{GMH}$. Asterisk indicates $p<0.05$ vs naive, number sign indicates $p<0.05$ vs $12 \mathrm{~h}$, dollar sign indicates $p<0.05$ vs 1 day, one-way ANOVA, Tukey's test, $n=6 /$ group. Data is presented as mean \pm SD

of rh-NT-4 were administered at $1 \mathrm{~h}, 25 \mathrm{~h}$, and $49 \mathrm{~h}$ after GMH. As shown in Fig. 2, pups in the vehicle group performed worse than the sham group in the short-term neurobehavioral tests, namely negative geotaxis test and righting reflex test, during the first 3 days after $\mathrm{GMH}$ (Fig. 2a, b, c). The administration of rh-NT-4(0.1 mg/kg) significantly improved performance in these two neurobehavioral tests compared with the vehicle group and low dose $(0.03 \mathrm{mg} / \mathrm{kg})$ group. The high dose treatment $(0.3 \mathrm{mg} / \mathrm{kg})$ had similar therapeutic effect to the medium dose $(0.1 \mathrm{mg} / \mathrm{kg})$. Therefore, the medium dose of rh-NT4 was chosen for long-term study and mechanism study.

\section{Exogenous NT-4 increased in brain tissue after intranasal administration}

To verify the sufficient exogenous NT-4 delivery via intranasal administrations into the brain, we used Western blot to test the concentration of human NT-4 at $6 \mathrm{~h}$ in sham $(n=6), \mathrm{GMH}+$ vehicle $(n=6)$, and GMH + rh-NT-4 $(n$ =6) groups. Anti-human NT-4 antibody was used in this part. As shown in the blot, the concentration of human NT-4 was significantly increased in the treatment group compared with sham and GMH + vehicle groups (Fig. 3a). Brain samples with schematic illustration showing the area in the perihematomal region (indicated by red square) were taken for immunofluorescence staining (Fig. 3b). Double immunofluorescence staining was performed to detect the co-localization of TrkB on microglia (Iba-1), neurons (NeuN), and astrocytes (GFAP) on day 3 after GMH (Fig. 3c). These images showed that TrkB was primarily expressed on microglia and neurons. And we also quantified the number of $\operatorname{TrkB}(-)$ and $\operatorname{TrkB}(-)$ microglia, neurons, and astrocytes, the results showed that $\operatorname{TrkB}(+)$ neurons and microglia are more than $\operatorname{TrkB}(-)$ neurons and microglia (Supplementary Fig. 4).

\section{Rh-NT-4 treatment ameliorated long-term neurological deficits after GMH}

To investigate the effects of rh-NT-4 treatment on the long-term neurological impairment induced by $\mathrm{GMH}$, neurological functions were assessed by rotarod test, foot fault test, and water maze at 4 weeks post GMH. RhNT-4 treatment significantly increased the falling latency at both 5 RPM and 10 RPM acceleration groups compared with the vehicle group (Fig. 4b, c). In the foot fault test, we found that vehicle animals had significantly more foot slips compared to sham animals, and the rhNT-4 treatment significantly improved the performance (Fig. 4a). In the water maze test, vehicle-treated animals spent more time finding the platform and had less time 


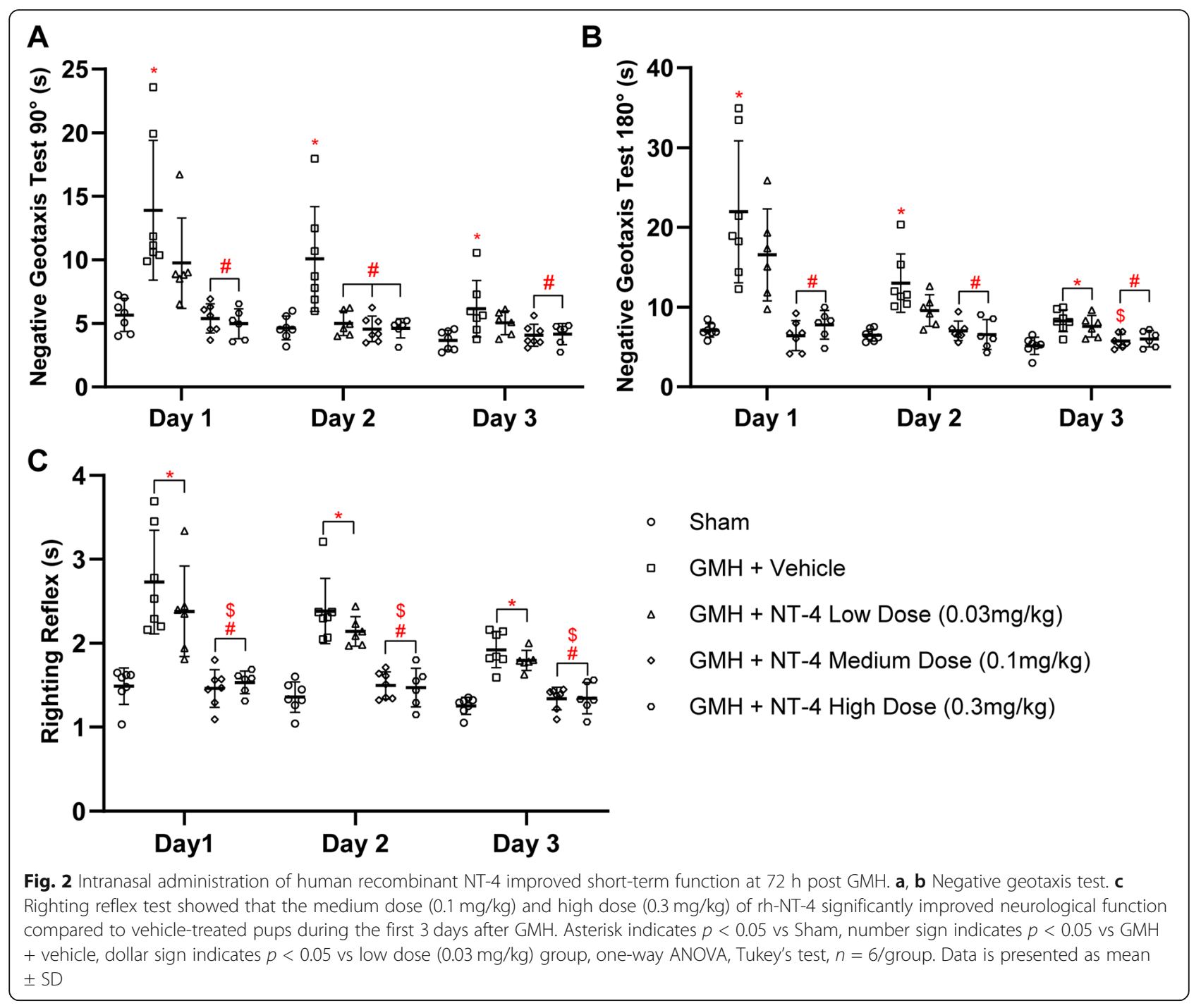

in the target quadrant compared with the sham group, suggesting that pups in the vehicle group had more severe cognitive impairment (Fig. 4g, h). Rh-NT-4-treated animals performed significantly better than vehicletreated animals (Fig. 4e, f). Meanwhile, there is no significant difference in swimming velocity (Fig. 4d) between these three groups, which means that longer escape latency was primarily due to spatial memory impairment, not slower velocity.

Rh-NT-4 treatment improves long-term brain morphology Ventricular dilation is the major complication of GMH. Thus, we used Nissl staining to assess the brain morphology at 28 days post GMH to see whether the rh-NT-4 treatment attenuated the long-term ventricular dilation (Fig. 5a). As shown in Fig. 5, the ventricular volume was significantly larger in the vehicle group compared with the sham group, and the rh-NT-4 treatment attenuated ventricular dilation (Fig. 5b). Cortical thickness (Fig. 5c), white matter loss (Fig. 5d), and gray matter loss (Fig. 5e) were presented as a ratio to the mean of the sham group. rh-NT-4 treatment significantly reduced these brain tissue losses compared with the vehicle group.

ANA-12 (a selective TrkB antagonist), LY294002 (a PI3K inhibitor), and FoxO1 Activation CRISPR reversed the protective effects of rh-NT-4 after GMH

In this part, we used the IL-6 as the marker of inflammation. According to the outcome study, the medium dose of rh-NT-4 $(0.1 \mathrm{mg} / \mathrm{kg})$ was administered at $1 \mathrm{~h}, 25$ $\mathrm{h}$, and $49 \mathrm{~h}$ post GMH. The samples were collected on day 3 post GMH. In the vehicle group, the phosphorylated TrkB increased while its downstream factors PI3K and phosphorylated Akt both decreased. FoxO1 and IL6 increased compared with the sham group. With the treatment of rh-NT-4, phosphorylated TrkB significantly increased compared with the vehicle group, and the expression of PI3K and phosphorylated Akt increased to 


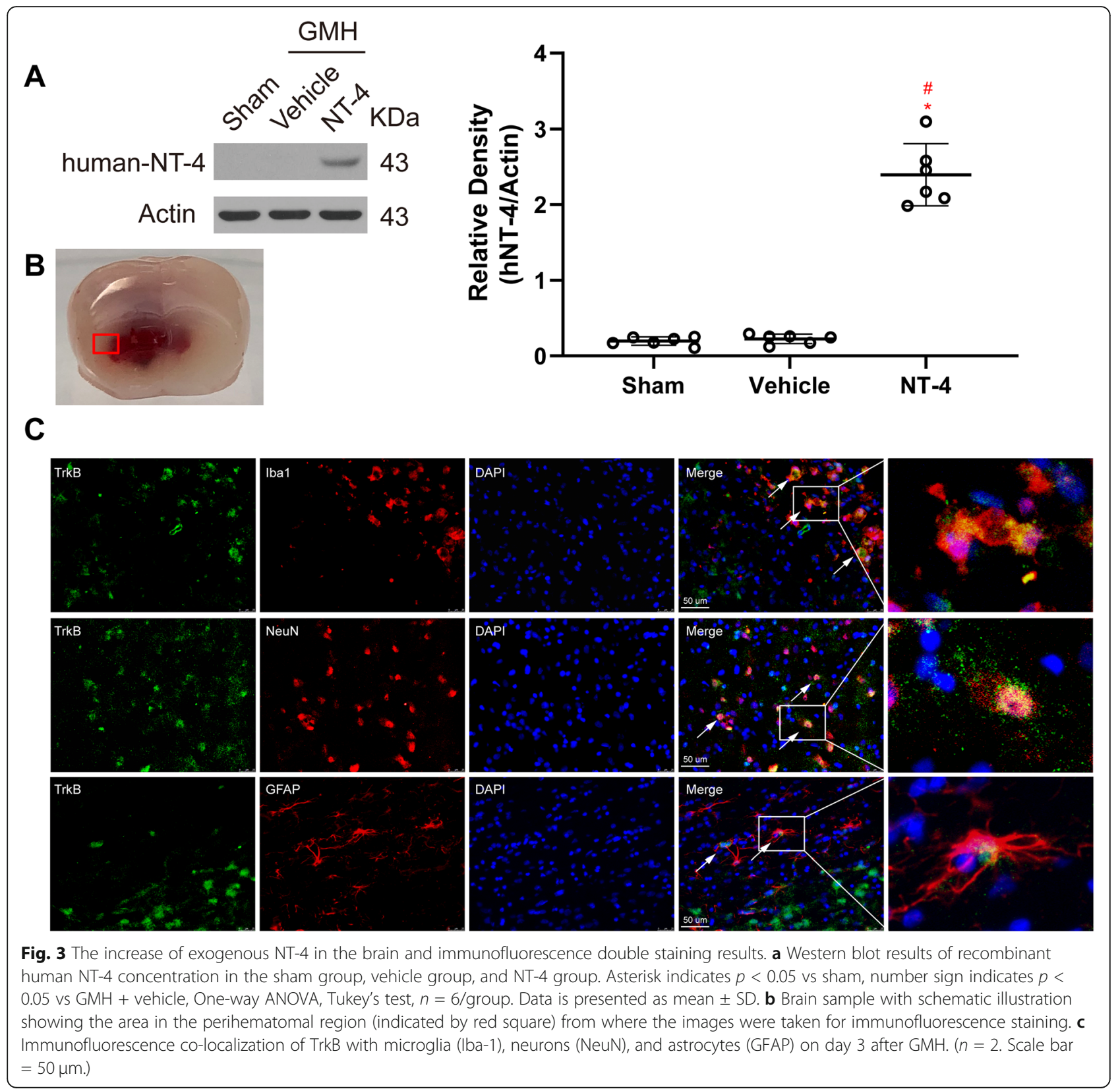

the base level which was similar to the sham group. The downstream factors, such as FoxO1 and IL-6, also decreased compared with the vehicle group. Thus, the ultimate effect of rh-NT-4 treatment was suppressing the expression of inflammatory cytokines, such as IL-6 (Figs. $6 a$ and $7 a)$. With the co-administration with ANA-12, the expression of phosphorylated TrkB significantly decreased compared with the treatment group. However, its downstream factors were unchanged compared with the vehicle group (Fig. 6b-g). In the LY294002 group, the elevated level of phosphorylated TrkB did not increase the expression of PI3K and phosphorylated Akt because of the inhibition of PI3K. Also, the downstream proteins FoxO1 and IL-6 were unchanged (Fig. 6b-g). FoxO1 activating CRISPR was also used to verify the pathway. Although rh-NT-4 treatment increased the expression of phosphorylated TrkB, PI3K and phosphorylated Akt, the FoxO1 expression was increased with the CRISPR (Fig. 7b-g), abolishing the therapeutic effect of rh-NT-4.

\section{Discussion}

$\mathrm{GMH}$ is the most common and devastating neurological injury of premature infants between 24-32 weeks of gestation [18]. A previous publication indicated that P7 SD rats are comparable to 32 weeks of human gestational 

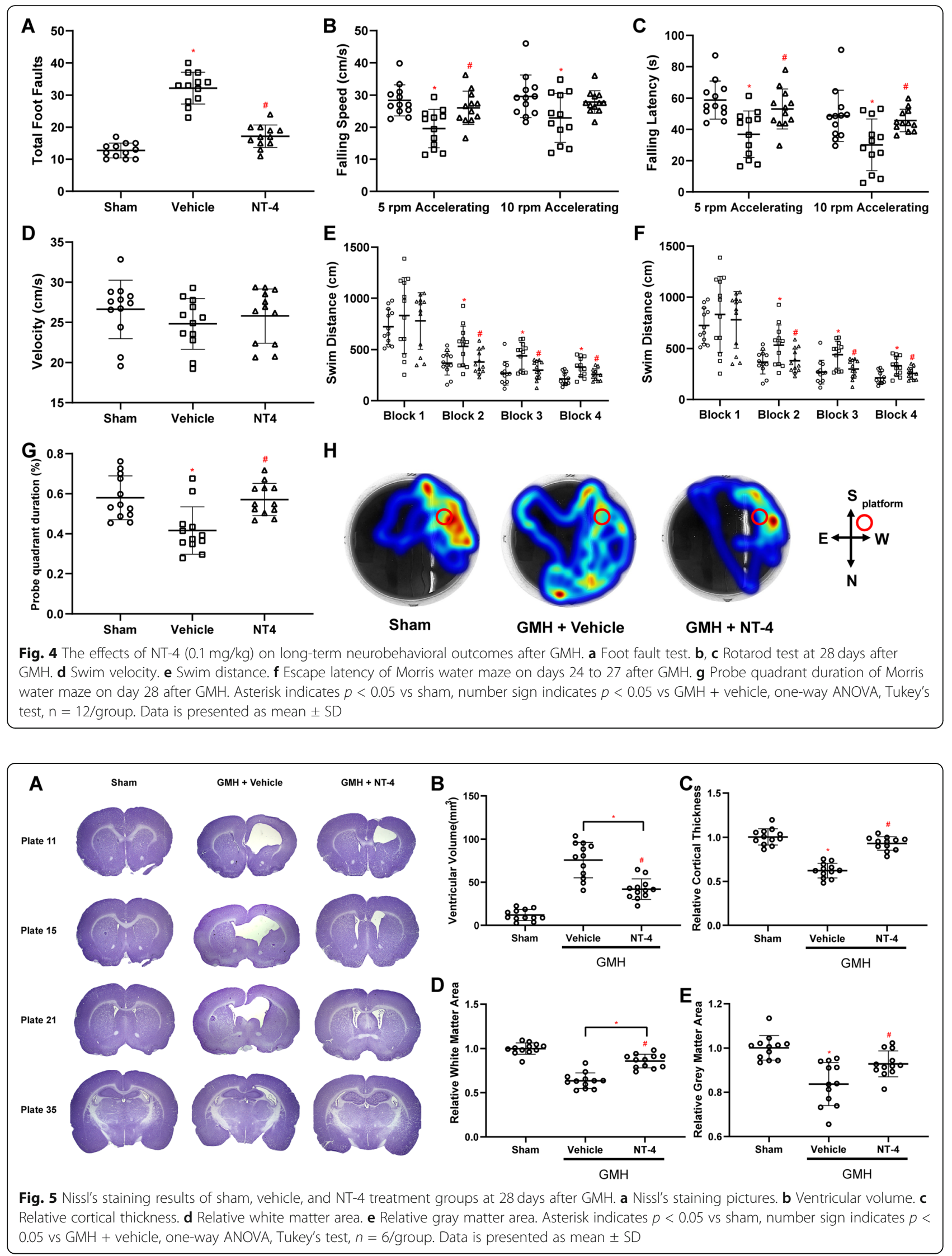


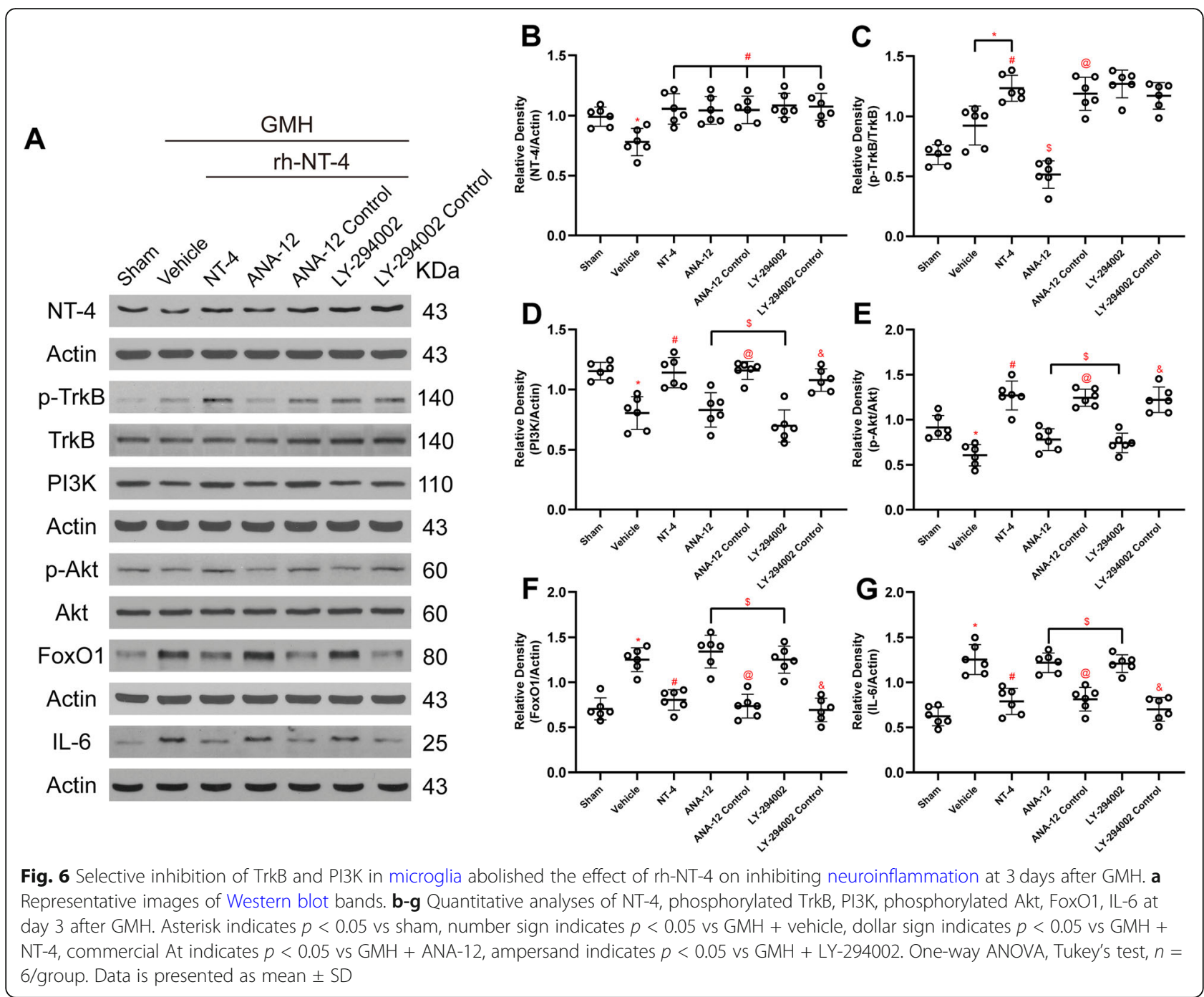

age. It is a devastating neonatal stroke characterized as neuroinflammation, hydrocephalus, primary and secondary brain injury, and developmental delay. Neuroinflammation is a trigger of secondary brain injury after GMH. Previous studies showed that NT-4 and BDNF are the high-affinity ligands of TrkB, and the phosphorylation of TrkB by BDNF can attenuate brain injury in stroke [13, 14]. In the present study, we investigated the potential anti-inflammatory effects of NT-4 binding to TrkB and its underlying mechanism after collagenase-induced GMH in rat pups. The results showed that the activation of TrkB with its ligand NT-4 improved the neurobehavioral functions, decreased the expression of proinflammatory cytokines, and improved long-term brain morphology. Mechanistically, administration of rh-NT-4 was associated with the upregulation of phosphorylated TrkB, PI3K and phosphorylated Akt, and downregulation of FoxO1 and IL-6 after GMH [1, 39].
In the first part of this study, we demonstrated that the expression of endogenous NT-4 was decreased after GMH induction, and then increased to base level on day 3.The expression of TrkB keep a relatively stable level in brain tissue during GMH. Phosphorylated TrkB increased in the early phase after GMH. According to the Western blot results in Fig. 1, the concentration of endogenous NT-4 was at a relative high level before GMH occurred, and the phosphorylated TrkB level was at a low level. We deduced that the endogenous NT-4 was initially consumed to phosphorylate TrkB to mitigate neuroinflammation after GMH. Furthermore, a higher level of endogenous NT-4 kept activating TrkB in order to exert its anti-inflammatory function. Phosphorylation is considered a transient process, not a lasting process. Therefore, we try to complement the endogenous NT-4 with rh-NT-4 in the early phase after GMH to keep TrkB activated. 


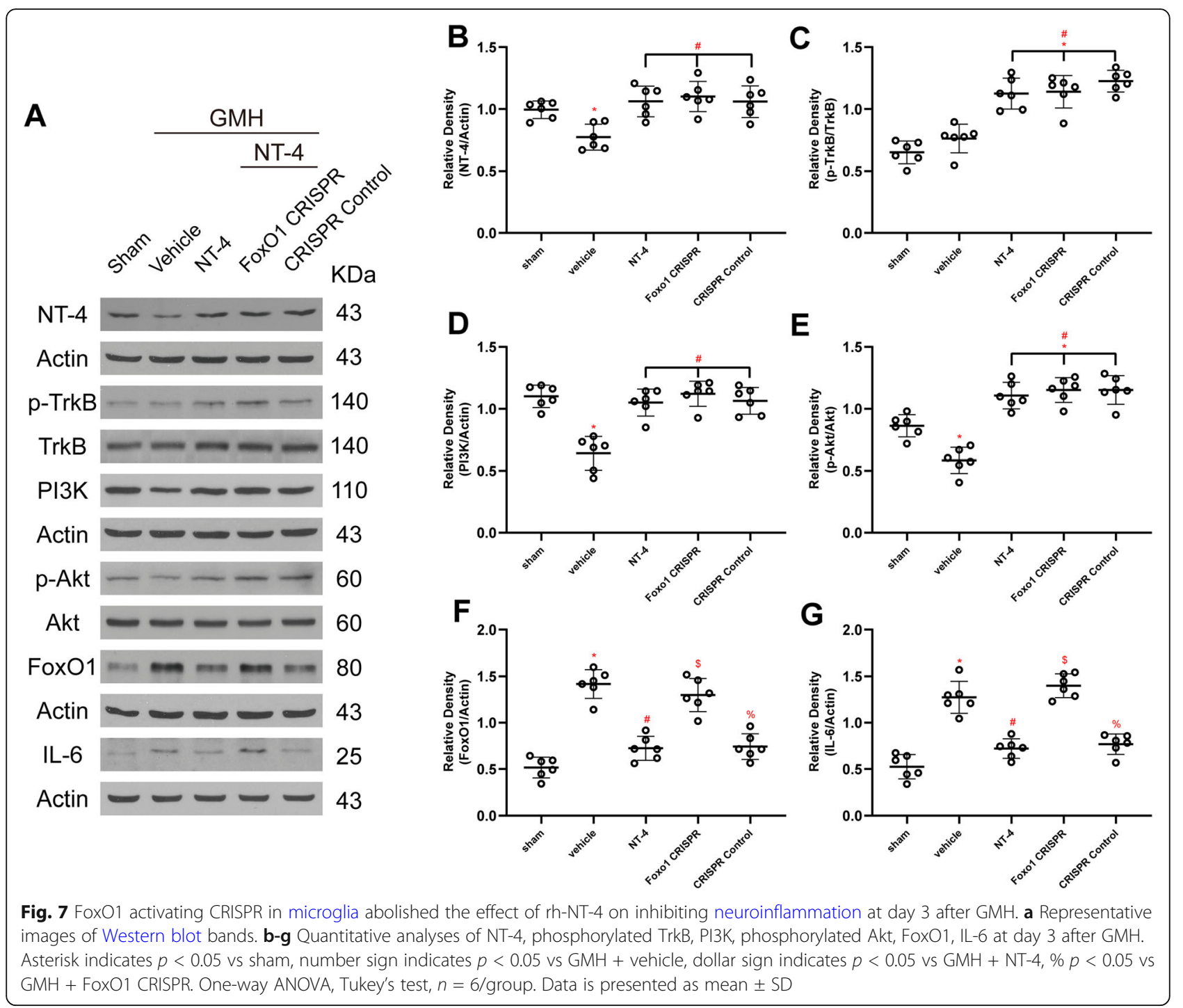

We did a negative geotaxis test and righting reflex test during the first 3 days after GMH in our outcome study. As shown in Fig. 2, the GMH + vehicle group demonstrated worse body reflex compared with the sham group. We gave three different doses of recombinant human NT-4 to determine the best dose for further studies. According to the data, the medium dose $(0.1 \mathrm{mg} / \mathrm{kg} /$ day) and high dose $(0.3 \mathrm{mg} / \mathrm{kg} /$ day $)$ treatments significantly improved the neurological outcomes after GMH, while the low dose $(0.03 \mathrm{mg} / \mathrm{kg} /$ day $)$ group performed worse than these two groups. Based on the short-term behavioral tests, we chose medium dose $(0.1 \mathrm{mg} / \mathrm{kg} /$ day $)$ for the long-term study and mechanism study.

Furthermore, we have also tested whether the recombinant human NT-4 was successfully delivered into the brain tissue via intranasal administration. A previous study showed that NT-4 levels in the brain and spinal cord were increased after intranasal administration, and it may have potential neuroprotective effects when used in models of CNS injuries [2]. To verify this, we used the anti-human-NT-4 antibody which cannot react with rats or mouse protein. The Western blot results showed that the expression of human-NT-4 significantly increased in the medium dose $(0.1 \mathrm{mg} /$ $\mathrm{kg} /$ day) group at 3 days after GMH compared with sham and GMH + vehicle groups. According to this, we ensured that the intranasal administration of rhNT-4 was successful.

In the outcome study, we also did the immunofluorescence double staining of TrkB with neurons (NeuN), microglia (Iba-1), and astrocytes (GFAP) to investigate the receptor localization. As shown in the immunofluorescence images, the majority of TrkB co-localized with microglia and neurons, and some TrkB receptors colocalized with astrocytes. In this study, we aimed to focus on the TrkB function on microglia. 
In the long-term behavioral study, we chose the medium dose of rh-NT-4 $(0.1 \mathrm{mg} / \mathrm{kg} /$ day $\times 7$ days $)$ for the treatment group to assess its therapeutic effects on day 28. In this part, we did a foot fault test, rotarod test, and water maze test to evaluate the differences between sham, GMH + vehicle, and GMH + rh-NT-4 groups. The foot fault test and rotarod test were performed to test the motor function at 28 days post GMH. Morris water maze test was performed to test the cognitive impairment in memory function. These tests indicated that the GMH + vehicle group showed worse motor function and cognition, and that the rh-NT-4 treatment attenuated this impairment.

Ventricular dilation is a common long-term outcome of GMH [23]. Recent studies have demonstrated that the inflammatory response in the choroid plexus results in the post-hemorrhagic ventricular dilation [20]. As shown in the Nissl staining results, rh-NT-4 treatment not only reduced the ventricular dilation but also ameliorated gray matter loss, white matter loss, and cortical thickness impairment. Based on these results, the activation of TrkB may ameliorate hydrocephalus and the mechanism might be due to the reduced microglia activation and pro-inflammatory cytokine generation. Collectively, TrkB activation on microglia may play an important role in the development of hydrocephalus, and the effect of NT-4 in reducing ventricular dilation may due to the suppression of pro-inflammatory cytokine release.

In this study, we focused on the effects of NT-4 mainly mediated by microglia signaling in GMH. Based on the mechanism study results, it is deducible that the PI3K/ Akt/FoxO1 pathway, which was activated by TrkB activation, may be an important signaling pathway to suppress microglia from generating pro-inflammatory cytokines, such as IL-6. Alternate pathways of NT-4 and TrkB were not evaluated in this study. Moreover, the immunofluorescence double staining indicated that the receptor TrkB was also expressed on neurons. Thus, we cannot eliminate the effects of NT-4 on neuron. As we all know, microglia are the resident macrophages of the brain and spinal cord, and they act as the first and main form of active immune defense in the CNS [26, 31]. Thus, it is deducible that microglial NT-4/TrkB signaling played a primary role in this neuroinflammation study. However, other effects of NT-4 directly on neurons are worthy of further investigation.

Previous studies indicated that NT-4 may be the preferable TrkB ligand as there would be less downregulation of TrkB with prolonged systemic treatments compared to BDNF [7, 21, 33]. However, these two ligands both have high affinity to TrkB. There might be different functions or effects between these two ligands, and this could be one of the weaknesses of this study. It would be more convincing if we compared the different therapeutic effects of these two ligands in the same pathway. We focused on the anti-inflammatory function of TrkB in microglia in this study. However, there are still many unknown functions of NT-4/TrkB pathway. Whether TrkB can express on lymphoid or myeloid cells in GMH model. What role does TrkB play in neuron and astrocytes after GMH. These issues need further research.

\section{Conclusions}

Our study demonstrated that recombinant human NT-4 reduced the expression of pro-inflammatory cytokines and attenuated neurological deficits in rat pups after GMH. The neuroprotective effects of rh-NT-4 are associated with TrkB/PI3K/Akt/FoxO1 pathway. These results indicated that NT-4 could be a promising therapeutic target to ameliorate neuroinflammation and hydrocephalus after GMH or other similar brain injuries.

\section{Supplementary information}

Supplementary information accompanies this paper at https://doi.org/10. 1186/s12974-020-01835-Z.

Additional file 1 :Fig S1. Summary of animal use, experimental groups and mortality rate in this study.

Additional file $\mathbf{2}$ Fig S2. Summary of proposal pathway. The interventions are marked in TrkB/PI3K/FoxO1 pathway in this study.

Additional file 3 Fig S3. Gel scans of all proteins in this study.

Additional file 4 Fig S4. Quantity of TrkB(-) and TrkB(-) microglia, neurons and astrocytes in CNS by immunofluorescence.

\section{Abbreviations}

Akt: Protein kinase B; BDNF: Brain-derived neurotrophic factor; CNS: Central nervous system; FoxO1: Forkhead box protein O1; GMH: Germinal matrix hemorrhage; GFAP: Glial fibrillary acidic protein; Iba-1: Ionized calciumbinding adaptor molecule; NT-4: Neurotrophin-4; NeuN: Neuronal specific nuclear protein; PI3K: phosphoinositide 3-kinase; TrkB: Tropomyosin receptor kinase $B$

\section{Acknowledgements}

Not applicable.

\section{Authors' contributions}

Yan Ding and Peng Li worked on the experimental design. Tianyi Wang and Junyi Zhang conducted the experiments, analyzed the data, and drafted the manuscript. Flores Jerry and Yan Ding worked on the manuscript revision. John H Zhang, Gang Chen, and Jiping Tang participated in the experimental design, data analysis and interpretation, and manuscript preparation. The authors read and approved the final manuscript.

\section{Funding}

This study was supported by the research grant R01-NS103822 from National Institute of Health to Dr. John H. Zhang.

\section{Availability of data and materials}

The authors confirm that all data underlying the findings are fully available without restriction. All relevant data are within the paper and its supporting information files.

\section{Ethics approval}

Humans were not used in this study. All animal experiments were approved by the Loma Linda University Institutional Animal Care and Use Committee. 


\section{Consent for publication}

Not applicable.

\section{Competing interests}

The authors declare that they have no competing interests.

Received: 5 December 2019 Accepted: 3 May 2020

Published online: 16 May 2020

\section{References}

1. Abbasi M, et al. Regulation of brain-derived neurotrophic factor and growth factor signaling pathways by tyrosine phosphatase Shp2 in the retina: a brief review. Front Cell Neurosci. 2018;12:85.

2. Alcala-Barraza SR, et al. Intranasal delivery of neurotrophic factors BDNF, CNTF, EPO, and NT-4 to the CNS. J Drug Target. 2010;18(3):179-90.

3. Anand SK, Mondal AC. TrkB receptor antagonism inhibits stab injury induced proliferative response in adult zebrafish (Danio rerio) brain. Neurosci Lett. 2018;672:28-33.

4. Aronowski J, Zhao X. Molecular pathophysiology of cerebral hemorrhage: secondary brain injury. Stroke. 2011;42(6):1781-6.

5. Ballabh P. Intraventricular hemorrhage in premature infants: mechanism of disease. Pediatr Res. 2010;67(1):1-8.

6. Ballabh P. Pathogenesis and prevention of intraventricular hemorrhage. Clin Perinatol. 2014;41(1):47-67.

7. Bothwell M. NGF, BDNF, NT3, and NT4. Handb Exp Pharmacol. 2014;220:315.

8. Chen $\mathrm{Q}$, et al. Simvastatin promotes hematoma absorption and reduces hydrocephalus following intraventricular hemorrhage in part by upregulating CD36. Transl Stroke Res. 2017;8(4):362-73.

9. Chen $\mathrm{S}$, et al. Activation of melanocortin receptor 4 with RO27-3225 attenuates neuroinflammation through AMPK/NNK/p38 MAPK pathway after intracerebral hemorrhage in mice. J Neuroinflammation. 2018;15(1):106.

10. Chung JY, et al. Increased expression of neurotrophin 4 following focal cerebral ischemia in adult rat brain with treadmill exercise. PLoS One. 2013; 8(3):e52461

11. Ding $\mathrm{Y}$, et al. Astrogliosis inhibition attenuates hydrocephalus by increasing cerebrospinal fluid reabsorption through the glymphatic system after germinal matrix hemorrhage. Exp Neurol. 2019;320:113003.

12. Dong $\mathrm{H}$, et al. Lithium ameliorates lipopolysaccharide-induced microglial activation via inhibition of toll-like receptor 4 expression by activating the PI3K/Akt/FoxO1 pathway. J Neuroinflammation. 2014;11:140.

13. Endres $\mathrm{M}$, et al. Ischemic brain damage in mice after selectively modifying BDNF or NT4 gene expression. J Cereb Blood Flow Metab. 2000;20(1):13944.

14. Endres $\mathrm{M}$, et al. Stroke damage in mice after knocking the neutrophin-4 gene into the brain-derived neurotrophic factor locus. J Cereb Blood Flow Metab. 2003;23(2):150-3.

15. Esmaeili $A$, et al. Alteration in messenger RNA neurotrophin 4 and tyrosine kinase receptors B expression levels following spinal cord injury. J Neurosurg Sci. 2018;62(2):146-52.

16. Fischer $\mathrm{DL}$, et al. Subthalamic nucleus deep brain stimulation employs trkB signaling for neuroprotection and functional restoration. J Neurosci. 2017; 37(28):6786-96.

17. Flores JJ, et al. PPARgamma-induced upregulation of CD36 enhances hematoma resolution and attenuates long-term neurological deficits after germinal matrix hemorrhage in neonatal rats. Neurobiol Dis. 2016;87:12433.

18. Georgiadis $\mathrm{P}$, et al. Characterization of acute brain injuries and neurobehavioral profiles in a rabbit model of germinal matrix hemorrhage Stroke. 2008;39(12):3378-88.

19. Heron M, et al. Annual summary of vital statistics: 2007. Pediatrics. 2010; 125(1):4-15.

20. Karimy JK, et al. Inflammation-dependent cerebrospinal fluid hypersecretion by the choroid plexus epithelium in posthemorrhagic hydrocephalus. Nat Med. 2017;23(8):997-1003.

21. Khan N, Smith MT. Neurotrophins and neuropathic pain: role in pathobiology. Molecules. 2015;20(6):10657-88.

22. Klebe $D$, et al. Acute and delayed deferoxamine treatment attenuates longterm sequelae after germinal matrix hemorrhage in neonatal rats. Stroke. 2014;45(8):2475-9.
23. Lekic T, et al. Rodent neonatal germinal matrix hemorrhage mimics the human brain injury, neurological consequences, and post-hemorrhagic hydrocephalus. Exp Neurol. 2012;236(1):69-78.

24. Li P, et al. Rh-IFN-alpha attenuates neuroinflammation and improves neurological function by inhibiting NF-kappaB through JAK1-STAT1/TRAF3 pathway in an experimental GMH rat model. Brain Behav Immun. 2019;79: $174-85$.

25. Li X, et al. Andrographolide ameliorates intracerebral hemorrhage induced secondary brain injury by inhibiting neuroinflammation induction. Neuropharmacology. 2018;141:305-15.

26. Lowe PP, et al. Reduced gut microbiome protects from alcohol-induced neuroinflammation and alters intestinal and brain inflammasome expression. J Neuroinflammation. 2018;15(1):298.

27. Lv O, et al. Mild hypothermia protects against early brain injury in rats following subarachnoid hemorrhage via the TrkB/ERK/CREB signaling pathway. Mol Med Rep. 2016;14(4):3901-7.

28. MacLellan $\mathrm{CL}$, et al. Intracerebral hemorrhage models in rat: comparing collagenase to blood infusion. J Cereb Blood Flow Metab. 2008;28(3):51625.

29. Mao XY, et al. Huperzine A alleviates oxidative glutamate toxicity in hippocampal HT22 cells via activating BDNF/TrkB-dependent PI3K/Akt/ mTOR signaling pathway. Cell Mol Neurobiol. 2016:36(6):915-25.

30. Matei $\mathrm{N}$, et al. Intranasal wnt3a attenuates neuronal apoptosis through Frz1/ PIWIL1a/FOXM1 pathway in MCAO rats. J Neurosci. 2018;38(30):6787-801.

31. Najafi AR, et al. A limited capacity for microglial repopulation in the adult brain. Glia. 2018

32. Nerurkar PV, et al. Momordica charantia (bitter melon) attenuates high-fat diet-associated oxidative stress and neuroinflammation. J Neuroinflammation. 2011;8:64.

33. Proenca CC, et al. Differential effects of BDNF and neurotrophin 4 (NT4) on endocytic sorting of TrkB receptors. J Neurochem. 2016;138(3):397-406.

34. Skaper SD. Neurotrophic factors: an overview. Methods Mol Biol. 2018;1727: $1-17$.

35. Suzuki $K$, et al. In vivo genome editing via CRISPR/Cas9 mediated homology-independent targeted integration. Nature. 2016;540(7631):144-9.

36. Tang J, et al. Neuroprotective role of an $\mathrm{N}$-acetyl serotonin derivative via activation of tropomyosin-related kinase receptor B after subarachnoid hemorrhage in a rat model. Neurobiol Dis. 2015;78:126-33.

37. Tao Y, et al. Cannabinoid receptor-2 stimulation suppresses neuroinflammation by regulating microglial M1/M2 polarization through the CAMP/PKA pathway in an experimental GMH rat model. Brain Behav Immun. 2016;58:118-29.

38. Xie Z, et al. Exendin-4 attenuates neuronal death via GLP-1R/PI3K/Akt pathway in early brain injury after subarachnoid hemorrhage in rats. Neuropharmacology. 2018;128:142-51.

39. Yan P, et al. Palmitic acid triggers cell apoptosis in RGC-5 retinal ganglion cells through the Akt/FoxO1 signaling pathway. Metab Brain Dis. 2017;32(2): 453-60.

40. Zhang Y, et al. Chemerin suppresses neuroinflammation and improves neurological recovery via CaMKK2/AMPK/Nrf2 pathway after germinal matrix hemorrhage in neonatal rats. Brain Behav Immun. 2018;70:179-93.

\section{Publisher's Note}

Springer Nature remains neutral with regard to jurisdictional claims in published maps and institutional affiliations.

Ready to submit your research? Choose BMC and benefit from:

- fast, convenient online submission

- thorough peer review by experienced researchers in your field

- rapid publication on acceptance

- support for research data, including large and complex data types

- gold Open Access which fosters wider collaboration and increased citations

- maximum visibility for your research: over $100 \mathrm{M}$ website views per year

At BMC, research is always in progress.

Learn more biomedcentral.com/submission 